\title{
Calculated properties of point defects in Be-doped GaN
}

\author{
C. D. Latham* and R. M. Nieminen \\ Laboratory of Physics, Helsinki University of Technology, P.O. Box 1100, FIN-02015 HUT, Finland \\ C. J. Fall and R. Jones \\ School of Physics, University of Exeter, Exeter EX4 4QL, United Kindom \\ S. Öberg \\ Department of Mathematics, Luleå University of Technology, SE-97187 Luleå, Sweden \\ P. R. Briddon \\ Physics Center, School of Natural Science, University of Newcastle upon Tyne, Newcastle NE1 7RU, United Kindom
}

(Received 6 September 2002; revised manuscript received 8 November 2002; published 9 May 2003)

\begin{abstract}
The properties of several point defects in hexagonal gallium nitride that can compensate beryllium shallow acceptors $\left(\mathrm{Be}_{\mathrm{Ga}}\right)$ are calculated using the AIMPRO method based on local density functional theory. $\mathrm{Be}_{\mathrm{Ga}}$ itself is predicted to have local vibrational modes (LVM's) very similar to magnesium acceptors. The highest frequency is about $663 \mathrm{~cm}^{-1}$. Consistent with other recent studies, we find that interstitial beryllium double donors and single-donor beryllium split interstitial pairs at gallium sites are very likely causes of compensation. The calculations predict that the split interstitial pairs possess three main LVM's at about 1041, 789, and $738 \mathrm{~cm}^{-1}$. Of these, the highest is very close to the experimental observation in Be-doped GaN. Although an oxygen donor at the nearest-neighboring site to a beryllium acceptor $\left(\mathrm{Be}_{\mathrm{Ga}} \mathrm{O}_{\mathrm{N}}\right)$ is also a prime suspect among defects that are possibly responsible for compensation, its highest frequency is calculated to be about $699 \mathrm{~cm}^{-1}$ and hence is not related in any way to the observed center. Another mode for this defect is estimated to be about $523 \mathrm{~cm}^{-1}$ and is localized on the $\mathrm{O}_{\mathrm{N}}$ atom. These two vibrations of $\mathrm{Be}_{\mathrm{Ga}}-\mathrm{O}_{\mathrm{N}}$ are thus equivalent to those for the isolated substitutional centers perturbed by the presence of their impurity partners.
\end{abstract}

DOI: 10.1103/PhysRevB.67.205206

PACS number(s): 61.72.Bb, 61.72.Ji, 61.72.Vv, 71.15.Nc

\section{INTRODUCTION}

Considerable progress has been made in recent years in the understanding of point defects, impurities, and intentional doping in AlN and GaN. The most significant of these are the defects responsible for the parasitic violet and yellow luminesence, which although having the same root cause, possess somewhat different properties in each material. Several competing models were initially proposed. ${ }^{1-4}$ Both theory and experiment eventually came to the conclusion, however, that cation vacancies were strongly implicated in the source of the luminescence and that in $\mathrm{GaN}$ it possibly involved an electronic transition between a shallow donor and a deep acceptor. ${ }^{5}$ Further theoretical modeling of the problem finally demonstrated that the donor-acceptor pair consisted of substitutional oxygen $\mathrm{O}_{\mathrm{N}}$ and cation vacancies $V_{\mathrm{Al}}$ or $V_{\mathrm{Ga}} \cdot{ }^{6}$ These defects can also bind to one another forming $V_{\mathrm{Ga}}-\mathrm{O}_{\mathrm{N}}$ and $V_{\mathrm{Al}}-\mathrm{O}_{\mathrm{N}}$ complexes.

A crucial point is that the presence of $\mathrm{O}_{\mathrm{N}}$ donors actually provides the thermodynamic driving force for the creation of the cation vacancies. Both $V_{\mathrm{Al}}$ and $V_{\mathrm{Ga}}$ are triple acceptors, and once ionized their formation energy falls steeply as the chemical potential rises. Experimental evidence for this is provided by the observation that the yellow luminescence in $\mathrm{GaN}$ is suppressed in $p$-type crystals heavily doped with magnesium: these contain $\mathrm{Mg}$ acceptors $\mathrm{Mg}_{\mathrm{Ga}}$ which lowers the chemical potential in the band gap. ${ }^{7-9}$ Gallium vacancies have also been observed directly by positron annihilation spectroscopy. ${ }^{10,11}$ Their concentration $\left[V_{\mathrm{Ga}}\right]$ is both directly proportional to the measured intensity of the yellow lumines- ence and falls linearly with increasing magnesium concentration $[\mathrm{Mg}]$. Magnesium acceptors are negatively charged centers, and positrons are bound to them in shallow hydrogenic states that are detected in these experiments. Another clear finding is that the measured positron lifetime is only consistent with the open volume being $V_{\mathrm{Ga}}$ and not nitrogen vacancies $V_{\mathrm{N}}$. The latter are both significantly smaller in size and are expected to be fairly shallow donors that are in a positive charge state, making them inoperative as positron traps. Moreover, the formation energy of $V_{\mathrm{N}}$ in $n$-type GaN is predicted to be much more than for $V_{\mathrm{Ga}}$ and is far too large for there to be any significant concentration present when the Fermi level lies anywhere from midgap upwards.

Additional information is provided by isochronal annealing studies, where the thermal treatment results in a reduction of the electrical resistivity of Mg-doped specimens of $\mathrm{GaN}$ by six orders of magnitude. ${ }^{12}$ This is attributed to the activation of $\mathrm{Mg}_{\mathrm{Ga}}$. It is suggested that $\mathrm{Mg}-\mathrm{H}$ complexes dissociate during this process, but photoluminescence and Hall effect measurements taken at each step during the experiments show that the mechanism is not at all straighforward. This is emphasized by the observation that the $3125 \mathrm{~cm}^{-1}$ local vibrational mode associated with $\mathrm{Mg}-\mathrm{H}$ complexes possesses an electric dipole aligned at $130 \pm 5^{\circ}$ to the $c$ axis of hexagonal GaN. ${ }^{13}$ It is difficult to reconcile this with the simplest models for the defect where a hydrogen atom is located at any high-symmetry site near to a $\mathrm{Mg}_{\mathrm{Ga}}$ atom: a reanalysis of the experimental results seems to be necessary. Theoretical investigations of $\mathrm{Mg}-\mathrm{H}$ complexes in 
cubic $\mathrm{GaN}$ predict that its structure consists of a $\mathrm{H}$ atom located at the antibonding site to one of the $\mathrm{N}$ atoms neighboring a substitutional $\mathrm{Mg}_{\mathrm{Ga}}$ atom. ${ }^{14}$ The localized nature of the chemical bonding means that it is reasonable to assume that the equivalent structure in hexagonal crystals would have very nearly the same formation energy.

The complex nature of point defects in $\mathrm{GaN}$ is further amplified by another investigation by photoluminescence spectroscopy and Hall effect measurements. ${ }^{15}$ Four samples doped with varying amounts of silicon $\left([\mathrm{Si}] \approx 2 \times 10^{17}-2\right.$ $\times 10^{19} \mathrm{~cm}^{-1}$ ) and one nominally undoped control were studied $\left([\mathrm{Si}] \approx 4 \times 10^{17} \mathrm{~cm}^{-1}\right)$. Both $\mathrm{Si}_{\mathrm{Ga}}$ donors and $\mathrm{Mg}_{\mathrm{Ga}}$ acceptors were detected in the reference specimen containing residual impurities. In addition to several sharper lines in the range $350-425 \mathrm{~nm}(3.6-3.0 \mathrm{eV})$, the photoluminescence spectrum of this sample exhibited the characteristic broadband yellow emission at about $550 \mathrm{~nm}(2.2 \mathrm{eV})$. A second, deeper donor was also present in the silicon-doped material and this was tentatively attributed to $\mathrm{O}_{\mathrm{N}}$.

Defects containing interstitial atoms have been the subject of more recent experimental investigations. ${ }^{16,17}$ In these studies, electrons with a kinetic energy of $2.5 \mathrm{MeV}$ were used to create centers in $\mathrm{GaN}$ that are paramagnetic. Their nature and behavior depended on whether the experiments were performed in situ at $4.2 \mathrm{~K}$ or at room temperature and on the subsequent thermal treatment of the samples. Optically detected electron paramagnetic resonance (ODEPR) signals exhibited well-resolved hyperfine lines that could clearly be identified as being due the presence of ${ }^{69} \mathrm{Ga}$ and ${ }^{71} \mathrm{Ga}$ isotopes in the centers. They were also correlated with various features in the photoluminescence spectra of the specimens studied. There is little doubt that gallium atoms displaced into several different interstitial configurations by the electron irradiation are responsible. In the special case of the experiments conducted in situ at $4.2 \mathrm{~K}$, it was quite clear that doubly ionized, interstitial gallium atoms $\mathrm{Ga}_{i}^{2+}$ were detected at either the $O$ or $T$ site in the wurtzite lattice. It was estimated from the annealing studies that these defects migrate with an activation energy $\sim 0.7 \mathrm{eV}$ and can be trapped in a complex that has a characteristic $0.88-\mathrm{eV}$ photoluminescence (PL) emission associated with it. The phonon sidebands in the PL spectrum of this center very closely resemble ones at $0.841 \mathrm{eV}$ in $\mathrm{GaP}$ that are known to involve $\mathrm{O}_{\mathrm{P}}$ donors. ${ }^{18}$ Oxygen is a common impurity in $\mathrm{GaN}$ too; therefore, it is natural to conclude that $\mathrm{O}_{\mathrm{N}}$ donors are in some way connected with the complex responsible for the $0.88-\mathrm{eV} \mathrm{PL}$ emission, in a form also involving intersitial gallium $\mathrm{Ga}_{i}$.

Beryllium is another candidate for a shallow acceptor in $\mathrm{GaN}$. Theoretical models based on the local density approximation (LDA) of density functional theory suggest that compensation due to interstitial atoms may be responsible for the very poor efficiency of $\mathrm{Be}$ as a $p$-type dopant. ${ }^{19}$ Recently it has been proposed that $\mathrm{Be}_{i}$ atoms migrate to $\mathrm{Be}_{\mathrm{Ga}}$ and form $(\mathrm{Be}-\mathrm{Be})_{\mathrm{Ga}}$ donors. ${ }^{20}$ The presence of these defects would also explain the failure to achieve $p$-type electrical activity in $\mathrm{GaN}$ doped with Be.

Although the $\mathrm{Be}_{\mathrm{Ga}}$ acceptor is apparently a simple defect, experimental and theoretical attempts to determine the energy of the acceptor level have not yet found a reliable value. ${ }^{20}$ The consensus is that $\mathrm{Be}_{\mathrm{Ga}}$ is shallower than $\mathrm{Mg}_{\mathrm{Ga}}$. For example, depending on the choice of parameters and approximations used, effective mass calculations predict the difference is about $10-15 \mathrm{meV} .{ }^{21}$ Another model that depends on the difference in electronegativity between a dopant and the atom it replaces predicts an even lower ionization energy for $\mathrm{Be}_{\mathrm{Ga}} \cdot{ }^{22}$

In this article, we present the results of total energy calculations for a number or Be-related centers in GaN. In particular, we report structures, formation energies, and local vibrational modes (LVM's), with the aim of elucidating the problem of compensation in GaN.

\section{METHOD}

The total energies of supercells are calculated using a method based on self-consistent local-density-functional theory, AIMPRO. Only a brief summary of the main points is given here: for a more detailed description see Refs. 23 and 24. A basis set of Gaussian orbitals is used to describe the Kohn-Sham wave functions of the valence electrons, where suitable multiplicative factors provide $s-, p$-, and optionally for each exponent, $d$-orbital symmetries. Even-tempered basis sets were generated and optimized by a similar procedure to that descibed in previous work on carbon doping in III-V semiconductors. ${ }^{25}$ Core electrons are replaced by a pseudopotential based on the Troullier-Martins scheme. ${ }^{26}$ This is modified by a nonlinear core correction (nlcc) to include approximately the effects of the Ga $3 d$ electrons without explicitly including them in the valence orbitals. ${ }^{27}$ The charge density is represented by a plane-wave basis in reciprocal space. An automatic procedure ensures that the number of shells of vectors $\mathbf{R}_{L}$ used to evaluate the Madelung energy is sufficient. We use the Monkhorst-Pack (MP) scheme to sample the band structure. ${ }^{28}$ Both the largest reciprocal lattice vector of the charge-density Fourier expansion and the mesh of $\mathbf{k}$ points are chosen so that the total energy $E_{\text {total }}$ is converged with respect to these parameters. MP $2 \times 2 \times 2$ k-point sampling is sufficient for structural relaxation, and calculation of LVM's. However, to achieve full convergence in the total energy to the level of $\$ 10^{-5} \mathrm{Ry}$, it is necessary to use MP $4 \times 4 \times 4$. When $\mathrm{O}$ atoms are present in a supercell, the cutoff for the charge-density Fourier expansion needs to be set to $220 \mathrm{Ry}$; otherwise, $\mathrm{N}$ atoms are the dominant factor, allowing this limit to be lowered to $150 \mathrm{Ry}$.

Defects are constructed in supercells with hexagonal crystal symmetry, where the ideal GaN structure contains 72 atoms. The forces acting on each atom are given by an analytical formula derived from the total energy expression. Structural optimization to minimize the total energy is performed by a conjugate-gradient algorithm. When a crystal contains defects its unit volume varies slightly from the ideal value by an amount that depends on the concentration of the defects and their relative size in the system. In small supercells the concentration of defects considered here is very much larger than in reality where it is close to the infinitely dilute limit; therefore, to simulate the true situation we choose to fix the lattice parameters describing supercells to the values that minimize the total energy of pure $\mathrm{GaN}$. If all 
the structural parameters of a pure $\mathrm{GaN}$ unit cell are optimized, the method reproduces the measured values with no significant error $(>1 \%)$. Local vibrational modes are calculated by using small displacements of selected atoms to generate a dynamical matrix numerically. If required, the Musgrave-Pople classical potential is used to obtain approximate second derivatives of energy to check whether including atoms for which $a b$ initio values are not available has any significant effect on the main LVM's of a defect. ${ }^{29,30}$ The parameters that describe the potential are fitted to LDA total energy calculations for a 72-atom supercell of pure GaN.

In order to establish a reasonable balance between speed and reliability for the calculations, many basis sets were constructed and tested. It has been found by trial and error that in most circumstances there is normally little to be gained in terms of minimizing total energies from using more than four Gaussian exponents per orbital, but that it is always necessary to include $d$ functions for the two smallest exponents in the basis set. The exceptions to this rule for the problems considered here are nitrogen in the form of isolated $\mathrm{N}_{2}$ molecules and oxygen atoms in defects. When a single, small molecule such as $\mathrm{N}_{2}$ is placed in a very large supercell, the wave function basis needs to be larger than normal. In the case of $\mathrm{N}_{2}$, it is neccessary to use a basis consisting of six Gaussian exponents per orbital with $d$ functions for the three smallest. The second special case is oxygen atoms in defects: these require five exponents with $d$ functions for the two smallest.

Formation energies are calculated by the conventional method as described by previous authors. ${ }^{20,31-33}$ In general terms, this is defined as

$$
E_{f}(q)=E_{d}(q)+q \mu_{e}-\sum_{i} n_{i} \mu_{i}
$$

where $E_{f}(q)$ is the formation energy of a defect in charge state $q, E_{d}(q)$ is the total energy of a supercell containing a defect, $n_{i}$ is the number of atoms of type $i, \mu_{i}$ is the chemical potential of atoms of type $i$, and $\mu_{e}$ is the electron chemical potential with respect to the top of the valence band of the pure material. The occupancy of defect-induced levels in the band gap of a semiconductor, and hence the charge state of defects, is determined by $\mu_{e}$. When $\mu_{e}$ is above a given defect level, the state is occupied; when $\mu_{e}$ is below the level it is unoccupied. Different charge states are simply achieved by setting the number of electrons in a supercell to the required quantity. When supercells contain charged defects, compensating corrections need to be applied; otherwise, the total energy would be divergent. This is achieved by adding a uniform background charge that cancels out the divergent terms in the total energy caused by the charged defects, including the dipole and quadrupole moments. ${ }^{34}$ A conservative estimate for the overall numerical error in $E_{f}(q)$ of the method is approximately $\pm 0.05 \mathrm{eV} .{ }^{24}$

The chemical potentials $\mu_{\mathrm{Ga}}$ and $\mu_{\mathrm{N}}$ depend on whether an excess of gallium or nitrogen is present during growth or annealing processes; hence orthorhombic Ga metal, diatomic $\mathrm{N}_{2}$ gas, and wurtzite $\mathrm{GaN}$ are the three relevant reference states. In other words, formation energies can vary over a

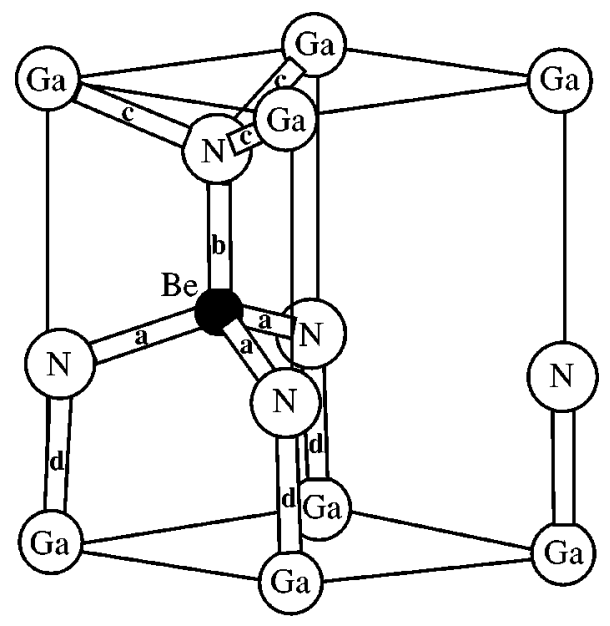

FIG. 1. Structure of a $\mathrm{Be}_{\mathrm{Ga}}$ acceptor: the lengths of the bonds marked $\mathbf{a}, \mathbf{b}, \mathbf{c}$, and $\mathbf{d}$ are given in Table I. A box is drawn around the structure to indicate its relationship with the hexagonal GaN crystal.

range of values limited by and with respect to these states depending on the conditions. Our calculations yield a value for the heat of formation $\Delta H_{f}=1.25 \mathrm{eV}$ for GaN from the elements in their standard states. To the accuracy of the method, this can be considered reasonably good agreement with the measured value $\Delta H_{f}=1.18 \mathrm{eV} .{ }^{35}$ The chemical potentials for impurity atoms are determined by the relevant stable compound. In the case of $\mathrm{Be}$ atoms, the reference states are hcp Be metal and $\alpha-\mathrm{Be}_{3} \mathrm{~N}_{2}$. The calculated heat of formation for $\alpha-\mathrm{Be}_{3} \mathrm{~N}_{2}$ from Be metal and $\mathrm{N}_{2}$ molecules is $\Delta H_{f}=7.04 \mathrm{eV}$. This also agrees reasonably well with the measured value $\Delta H_{f}=6.10 \mathrm{eV} .^{36}$

\section{RESULTS}

\section{A. $\mathrm{Be}_{\mathrm{Ga}}$ acceptor}

The starting point for these calculations is to calculate the structure of the $\mathrm{Be}_{\mathrm{Ga}}$ acceptor. In both the neutral and -1 charge states we find that the Be atom is located very close to the lattice site of the $\mathrm{Ga}$ atom that it replaces, and that there is an inward relaxation of the four neighboring $\mathrm{N}$ atoms; see Fig. 1 and Table I. The formation energy for the neutral defect in Ga-rich conditions is $E_{f}(0)=2.45 \mathrm{eV}$. Under $\mathrm{N}$-rich conditions the figure is smaller by $1 / 3$ of the heat of formation of $\mathrm{GaN}$, making $E_{f}(0)=2.03 \mathrm{eV}$.

To check whether the nlcc is a valid approximation, the calculations have been repeated in the neutral charge state using a very large basis that includes the Ga $3 d$ electrons explicitly. There is no significant difference in the outcome. Another crucial result is that the energy change associated with the structural relaxation resulting from ionization of the defect is $\lesssim 10 \mathrm{meV}$. This result differs significantly from those of Van de Walle et al. ${ }^{20}$ They find that the Be atom in the neutral defect is off center with a Be-N bond in the $c$ direction that is about $12 \%$ longer than a Ga-N bond in the bulk crystal. When the defect is ionized, this Be-N bond along the $c$ direction becomes about $5 \%$ shorter than a Ga-N bond, so that the Be atom is close to the lattice site. The 
TABLE I. Bond lengths $(\AA)$ for the $\mathrm{Be}_{\mathrm{Ga}}$ acceptor (and percentage change with respect to bulk GaN). The first column refers to whether Ga $3 d$ electrons are explicitly included in the basis or treated approximately by the nlcc. See also Fig. 1.

\begin{tabular}{lccccc}
\hline \hline Basis & Charge & N-Be $(\mathbf{a})$ & Be-N $(\mathbf{b})$ & N-Ga $(\mathbf{c})$ & Ga-N $(\mathbf{d})$ \\
\hline Ga $3 d$ & 0 & $1.797(-7.3 \%)$ & $1.775(-8.4 \%)$ & $1.929(-0.5 \%)$ & $1.933(-0.3 \%)$ \\
nlcc & 0 & $1.811(-7.5 \%)$ & $1.781(-9.0 \%)$ & $1.951(-0.4 \%)$ & $1.955(-0.2 \%)$ \\
nlcc & -1 & $1.805(-7.8 \%)$ & $1.802(-8.0 \%)$ & $1.943(-0.8 \%)$ & $1.954(-0.2 \%)$ \\
\hline \hline
\end{tabular}

energy of relaxation is $0.1 \mathrm{eV}$. We tried to reproduce this result by starting from structures in the neutral-charge state with a stretched Be-N along the $c$ axis, both for the nlcc basis and with Ga $3 d$ electrons included, but did not find even a local minimum for such a structure. Moreover, a search for an off-center structure using the popular VASP plane-wave pseudopotential method obtained nearly identical results to ours. ${ }^{37}$ Earlier calculations using 32-atom supercells are also in good agreement with our results. ${ }^{38-40}$

Calculating the energy of the acceptor level is more problematic. In addition to well-known shortcomings of density functional theory regarding excited states and an underestimation of band gaps, the total energies for charged supercells are rather sensitive to the details of the implementation used; therefore it is not very meaningful to provide absolute values for shallow levels. Moreover, in the case of shallow levels, these are delocalized states with a spatial extent much larger than the size of a supercell and thus are broadened into a "miniband" that makes the energy of the level even more ill defined. In the present calculations the width of the $\mathrm{Be}_{\mathrm{Ga}}$ state is about $0.4 \mathrm{eV}$. A more reliable quantity is the energy of a level relative to one for another defect. The ordering of levels is usually correct, although the absolute accuracy is low. In $\mathrm{GaN}$, as mentioned previously, it is thought that $\mathrm{Be}_{\mathrm{Ga}}$ is shallower than $\mathrm{Mg}_{\mathrm{Ga}}$. If this is the case, Be may be a more attractive dopant than $\mathrm{Mg}$ because the acceptor level for $\mathrm{Mg}_{\mathrm{Ga}}$ is not particularly shallow (208 $6 \mathrm{meV}$ ) (Ref. 41); hence the concentration of thermally activated carriers is relatively low at normal operating temperatures for devices. When $\mathrm{Mg}$ is replaced by $\mathrm{Be}$ in our calculations (and a full structural relaxation performed) the acceptor level — in terms of total energies, together with small corrections for shifts in the average potential - is about $0.09 \mathrm{eV}$ higher for $\mathrm{Mg}_{\mathrm{Ga}}$ than $\mathrm{Be}_{\mathrm{Ga}}$. This places the $\mathrm{Be}_{\mathrm{Ga}}$ level at approximately
$0.12 \mathrm{eV}$. Under Ga-rich conditions, the formation energy of the negatively charged state is, therefore, $E_{f}(-1)=(2.6$ $\left.-\mu_{e}\right) \mathrm{eV}$.

The highest-frequency local modes of atoms that are lighter than the host atom they replace are usually above the bulk lattice phonons. In Be-doped $\mathrm{GaN}\left([\mathrm{Be}] \gtrsim 10^{19} \mathrm{~cm}^{-3}\right)$ a local mode is observed by infrared spectroscopy well above the $A_{1}$ (LO, $735 \mathrm{~cm}^{-1}$ ) phonon frequency, at $789 \mathrm{~cm}^{-1}$, that is apparently in line with expectations for simple, substitional beryllium on a gallium lattice site $\mathrm{Be}_{\mathrm{Ga}} \cdot{ }^{42}$ However, this is probably not due to $\mathrm{Be}_{\mathrm{Ga}}$. The calculated frequencies all lie much lower, well below the $A_{1}$ lattice mode, and are comparable with those for $\mathrm{Mg}_{\mathrm{Ga}}$ - see Table II. In the case of $\mathrm{Mg}_{\mathrm{Ga}}$, both calculated and measured modes are given: there is very good agreement between theory and experiment. Not only are these modes obscured by the bulk phonons; they also possess $A_{1}$ symmetry and so would probably be too weak to be detected by infrared spectroscopy. ${ }^{42}$ In order to separate the local modes of the defect from the bulk phonons, second derivatives of energy for only the dopant atom and its four nearest neighbors $(\mathrm{N}$ atoms) are included in the dynamical matrix. If the next shells of Ga atoms are included, many bulklike modes associated with $\mathrm{Ga}-\mathrm{N}$ bonds appear in the spectrum. The collective motion of the $\mathrm{Ga}$ and $\mathrm{N}$ atoms swamps the vibrations localized on the dopant atom, and it becomes impossible to distinguish its modes. Note that the choice of basis and charge state has no significant effect on the calculated frequencies for $\mathrm{Be}_{\mathrm{Ga}}$.

The local modes of $\mathrm{Mg}_{\mathrm{Ga}}$ have been measured by Raman scattering experiments. ${ }^{43-46}$ It is quite possible that the same technique would also be able to observe the modes for $\mathrm{Be}_{\mathrm{Ga}}$ as they are so similar. These defects also have bands of nearly degenerate $E$ modes slightly below each of the $A_{1}$

TABLE II. Calculated and observed modes with $A$ symmetry for $\mathrm{Be}_{\mathrm{Ga}}$ and $\mathrm{Mg}_{\mathrm{Ga}}$ in $\mathrm{GaN}$.

\begin{tabular}{lccccc}
\hline \hline Description & \multicolumn{5}{l}{ Frequencies $\left(\mathrm{cm}^{-1}\right)$} \\
\hline $\mathrm{Be}_{\mathrm{Ga}}^{0}[$ AIMPRO (nlcc)] & - & 445 & 562 & 593 & 663 \\
$\mathrm{Be}_{\mathrm{Ga}}^{0}[$ AIMPRO $(\mathrm{Ga} \mathrm{3d})]$ & - & 446 & 580 & 594 & 669 \\
$\mathrm{Be}_{\mathrm{Ga}}^{-}[$AIMPRO (nlcc)] & - & 448 & 571 & 600 & 679 \\
\hline $\mathrm{Mg}_{\mathrm{Ga}}^{0}[$ AIMPRO (nlcc)] & - & - & 297 & 576 & 669 \\
$\mathrm{Mg}_{\mathrm{Ga}}\left[\right.$ Kaschner et al. $\left.{ }^{\text {a }}\right]$ & 136 & 262 & 320 & 595 & 656 \\
$\mathrm{Mg}_{\mathrm{Ga}}\left[\right.$ Harima et al. $\left.{ }^{\text {b }}\right]$ & - & 260 & - & - & 657 \\
\hline \hline
\end{tabular}

${ }^{\mathrm{a}}$ References 43 and 44 .

${ }^{\mathrm{b}}$ References 45 and 46 . 




FIG. 2. Structure of a single beryllium interstitial: the Be atom is equidistant from the nearest $\mathrm{N}$ atom shown in this view and the two equivalent ones in neighboring cells. The center of the hexagonal channel in the $c$ direction is marked by a dotted line. N.B. the $\mathrm{Ga}$ and $\mathrm{N}$ atoms are reversed with respect to Figs. 1 and 3.

frequencies. Modes of this character are not possible to classify further as mixing effects make any analysis meaningless.

The apparent discrepancy between the expected frequency for the highest $\mathrm{LVM}$ of $\mathrm{Be}_{\mathrm{Ga}}$, and the value calculated by the method used in this work can be understood as follows. Although $\mathrm{Be}$ is a light atom, it is also smaller than a $\mathrm{Ga}$ atom, and the bonds it makes with the four neighboring $\mathrm{N}$ atoms are $\approx 4 \%$ longer than Be-N bonds usually are. For example, in $\alpha-\mathrm{Be}_{3} \mathrm{~N}_{2}$, the average Be-N distance is $1.73 \AA$ (calculated). The longer a bond of a given type is, the weaker it becomes, and hence its characteristic frequency is lower.

\section{B. Interstitial beryllium}

Interstitial beryllium might reasonably be expected to exist as single atoms within the crystal, or as pairs of Be atoms occupying a Ga lattice site. The former has two valence electrons to donate, while the latter has one. Both types, therefore, should be compensating defects.

Figure 2 shows the relaxed structure for an isolated beryllium interstitial $\mathrm{Be}_{i}$; this is the lowest-energy structure found from several different initial configurations. The Be atom lies in the center of the hexagonal channel as viewed along the $c$ direction and about $0.30 \AA$ from the plane defined by the three neighboring $\mathrm{N}$ atoms. (Only one of these three equivalent neighbors is shown in Fig. 2.) These three $\mathrm{N}$ atoms are displaced from their normal positions towards the Be atom: the Be-N distance is about $1.69 \AA$. In the neutral state this defect is calculated to have a formation energy of $5.82 \mathrm{eV}$ (Ga rich). Removing one or two electrons from the system causes very little change to the structure. The $\mathrm{Be}_{i}$ donor level is found to be resonant with the conduction band. Therefore the defect would be autoionizing and is only stable in the +2 charge state. There are no states in the band gap. When $\mu$ $=0, \mathrm{Be}_{i}^{2+}$ is predicted to have a formation energy of about $-0.6 \mathrm{eV}$ (Ga rich). It should be remembered, however, that



FIG. 3. Structure of a beryllium split interstitial. The length of the central Be-Be bond marked $\mathbf{a}$ and neighboring $\mathrm{Be}-\mathrm{N}$ bonds marked $\mathbf{b}, \mathbf{c}, \mathbf{d}$, and $\mathbf{e}$ are given in Table III.

the band gap for GaN predicted by the LDA is only $2.2 \mathrm{eV}$ which is much less than the true value of $3.50 \mathrm{eV}^{47}$ This fact combined with uncertainties arising from the corrections needed to deal with charged supercells means that these energies cannot be considered particularly reliable.

Although a comprehensive survey of the energy surface has not been conducted, interstitial Be atoms are probably able to diffuse fairly easily though the crystal (recall that ODEPR experiments show that interstitial gallium ions $\mathrm{Ga}_{i}^{2+}$ are mobile well below room temperature). They might then be trapped by any substitutional Be atoms that they encounter if they are able to form split interstitial pair complexes $(\mathrm{Be}-\mathrm{Be})_{\mathrm{Ga}}$. Figure 3 shows the relaxed structure for $(\mathrm{Be}-\mathrm{Be})_{\mathrm{Ga}}$; both the neutral and +1 charge states of this defect are very similar (see also Table III). As with the single interstitial, this is the lowest-energy structure that was found from several different initial configurations. Under Ga-rich conditions, the formation energy of the neutral defect is $E_{f}(0)=4.08 \mathrm{eV}$. The donor level is found to lie in the conduction band; hence only the +1 charge state is stable. There are no states in the band gap. When $\mu=0, E_{f}(+1)$ $\approx 0.4 \mathrm{eV}$, though the same remarks concerning reliability of calculated energies of charged states as for $\mathrm{Be}_{i}$ apply here too. Figure 4 provides a summary of formation energies as a function of electron chemical potential for $\mathrm{Be}_{\mathrm{Ga}}, \mathrm{Be}_{i}$, and $(\mathrm{Be}-\mathrm{Be})_{\mathrm{Ga}}$ defects. From this it can be seen that the binding energy for the reaction $\mathrm{Be}_{i}+\mathrm{Be}_{\mathrm{Ga}} \rightarrow(\mathrm{Be}-\mathrm{Be})_{\mathrm{Ga}}$ and, when all the components are in the neutral state, is estimated to be approximately $4.2 \mathrm{eV}$. This figure falls to about $1.5 \mathrm{eV}$ when $\mu_{e}=0$ and the defects are in their stable charge states.

TABLE III. Bond lengths $(\AA)$ for the $(\mathrm{Be}-\mathrm{Be})_{\mathrm{Ga}}$ defect in its neutral and ionized states. See also Fig. 3.

\begin{tabular}{lccccc}
\hline \hline Charge & $\mathrm{Be}-\mathrm{Be}(\mathbf{a})$ & $\mathrm{N}-\mathrm{Be}(\mathbf{b})$ & $\mathrm{N}-\mathrm{Be}(\mathbf{c})$ & $\mathrm{Be}-\mathrm{N}(\mathbf{d})$ & $\mathrm{Be}-\mathrm{N}(\mathbf{e})$ \\
\hline 0 & 1.880 & 1.569 & 1.653 & 1.739 & 1.837 \\
+1 & 1.886 & 1.571 & 1.651 & 1.744 & 1.847 \\
\hline \hline
\end{tabular}



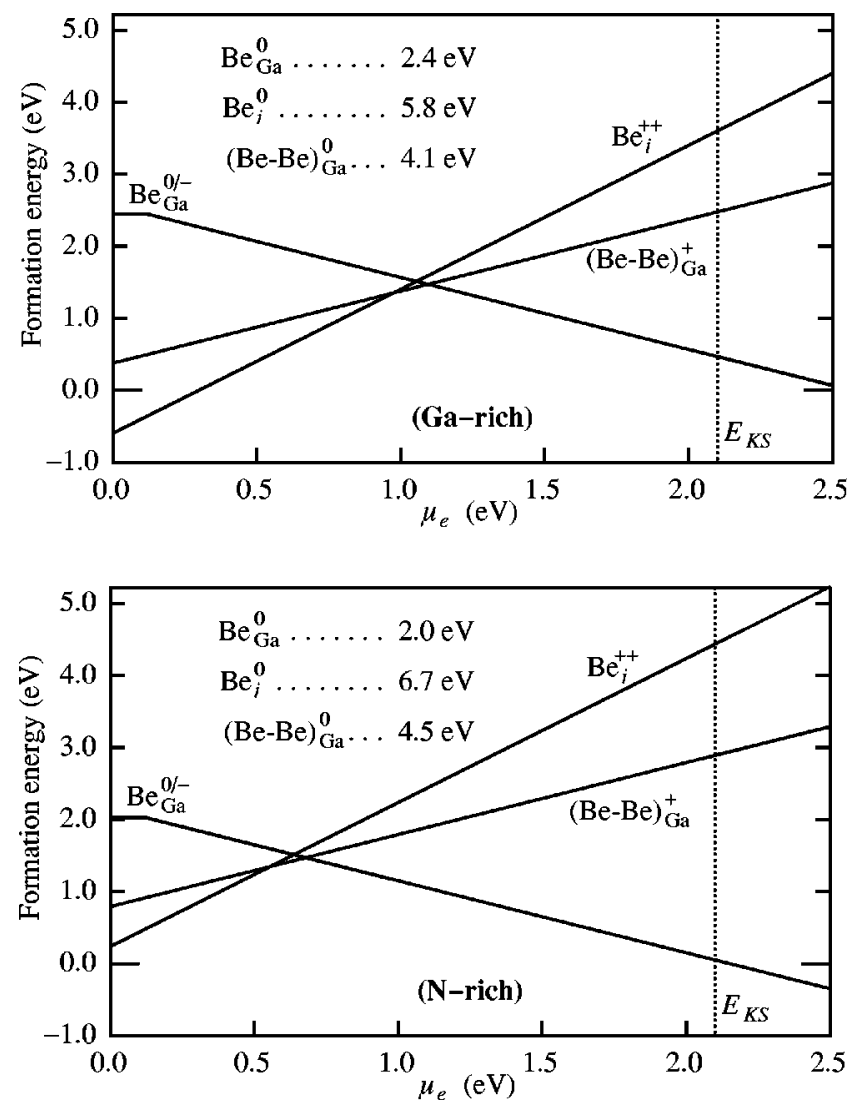

FIG. 4. Formation energy $E_{f}(q)$ plotted as a function of $\mu_{e}$ for $\mathrm{Be}_{\mathrm{Ga}}, \mathrm{Be}_{i}^{2+}$, and $(\mathrm{Be}-\mathrm{Be})_{\mathrm{Ga}}^{+}$defects in $\mathrm{GaN}$ under Ga-rich (upper) and N-rich (lower) conditions. The vertical dotted line marked $E_{\mathrm{KS}}$ at $\mu_{e} \approx 2.2 \mathrm{eV}$ represents the Kohn-Sham band gap of the LDA. The kink in the line for $\mathrm{Be}_{\mathrm{Ga}}^{0 /-}$ at $\mu_{e} \approx 0.1 \mathrm{eV}$ is caused by the change in charge state of the defect between 0 and -1 at this point.

Although the estimates for formation and ionization energies of the defects considered so far are only approximate, they do at least indicate that in material doped with high concentrations of beryllium, a substantial fraction of it may be expected to be in the form of interstitial defects. Of the two types, the split pair appears to be the more suitable candidate for detection by infrared LVM spectroscopy or Raman scattering experiments. The three highest calculated modes for the $(\mathrm{Be}-\mathrm{Be})_{\mathrm{Ga}}$ center are well above the bulk phonons: their frequencies are estimated to be 1041, 789, and $738 \mathrm{~cm}^{-1}$. These values are obtained using ab initio second derivatives of energy for the two $\mathrm{Be}$ atoms and eight of the nearest neighbors surrounding the center only. If the dynamical matrix is extended to include all atoms in the supercell by using the Musgrave-Pople potential to estimate the second derivatives of energy for which ab initio values are not available, the frequencies shift only by a few wave numbers to 1042,793 , and $744 \mathrm{~cm}^{-1}$. This demonstrates both that sufficient $a b$ initio derivatives have been calculated and that these are local modes of the defect. The highest of the three modes is a stretching motion of the Be-Be bond, while the other two are essentially local stretch-bend oscillations localized on each of the two Be atoms. As the structure of the defect is so similar in both the neutral and +1 charge states, the frequencies of their modes are equal to within $\sim 1 \mathrm{~cm}^{-1}$.

In addition to the $789 \mathrm{~cm}^{-1}$ mode mentioned previously, two high-frequency lines have been observed in Be-doped $\mathrm{GaN}$ at 1055 and $1063 \mathrm{~cm}^{-1} \cdot{ }^{42}$ It is tempting to assign one of the high-frequency pair to the Be-Be stretch mode at $1041 \mathrm{~cm}^{-1}$ in the calculations, while there is a rather obvious match at $789 \mathrm{~cm}^{-1}$. As the material contains a high concentration of oxygen, the weaker of the two high-frequency signals with a frequency of $1063 \mathrm{~cm}^{-1}$ could simply be due to a variant of the $(\mathrm{Be}-\mathrm{Be})_{\mathrm{Ga}}$ complex with a substitional $\mathrm{O}_{\mathrm{N}}$ neighbor. A problem with these assignments is that while the lines observed at 1055 and $1063 \mathrm{~cm}^{-1}$ are correlated in terms of signal strength, they do not appear to be related to the $789 \mathrm{~cm}^{-1}$ mode. ${ }^{42}$ Therefore, it is probably safe to accept the model for the high-frequency pair, but the signal at $789 \mathrm{~cm}^{-1}$, on balance, seems to require a different explanation.

\section{C. $\mathrm{Be}_{\mathrm{Ga}}-\mathrm{O}_{\mathrm{N}}$}

One alternative model for the observed modes that can safely be excluded is a $\mathrm{Be}_{\mathrm{Ga}}-\mathrm{O}_{\mathrm{N}}$ complex. It is very likely that this defect exists in $\mathrm{GaN}$ doped with Be due to the presence of significant concentrations of oxygen in the material. Moreover, we calculate that the binding energy of the $\mathrm{Be}_{\mathrm{Ga}}-\mathrm{O}_{\mathrm{N}}$ complex relative to its isolated components $\mathrm{Be}_{\mathrm{Ga}}^{+}$ and $\mathrm{O}_{\mathrm{N}}^{-}$is about $0.34 \mathrm{eV}$. This defect possesses two main modes with frequencies calculated to be 699 and $523 \mathrm{~cm}^{-1}$. The higher-frequency one is strongly localized on the $\mathrm{Be}$ atom, while the lower is almost entirely due to the O-atom. These, therefore, represent perturbed versions of isolated $\mathrm{Be}_{\mathrm{Ga}}$ and $\mathrm{O}_{\mathrm{N}}$. The measured frequency for $\mathrm{O}_{\mathrm{N}}$ is $544 \mathrm{~cm}^{-1} .{ }^{48}$ Figure 5 shows how the calculated LVM's for $\mathrm{Be}_{\mathrm{Ga}},(\mathrm{Be}-\mathrm{Be})_{\mathrm{Ga}}$, and $\mathrm{Be}_{\mathrm{Ga}}-\mathrm{O}_{\mathrm{N}}$ compare with the modes observed in heavily Be-doped GaN.

\section{SUMMARY AND CONCLUSIONS}

The results of our calculations provide more support for the idea that $\mathrm{Be}_{\mathrm{Ga}}$ is a shallower dopant than $\mathrm{Mg}_{\mathrm{Ga}}$ and has a low formation energy, but is strongly compensated by interstitial defects. GaN with a high $\mathrm{Be}$ content is semi-insulating. ${ }^{49,50}$ Although Be has a much smaller atomic mass than $\mathrm{Mg}$, the $\mathrm{Be}_{\mathrm{Ga}}$ defect has relatively long bonds to its four neighboring $\mathrm{N}$ atoms. This has the effect of lowering the frequency of its LVM's so that, by coincidence, its highest two modes are estimated to be very nearly equal to those for $\mathrm{Mg}_{\mathrm{Ga}}$. The LVM's of $\mathrm{Mg}_{\mathrm{Ga}}$ have been observed by Raman scattering, but not $\mathrm{Be}_{\mathrm{Ga}}$; hence we suggest that it may be possible to detect the LVM's of $\mathrm{Be}_{\mathrm{Ga}}$ by the same method.

Interstitial $\mathrm{Be}$ atoms may exist singly- $\mathrm{Be}_{i}$ - or in a split pair configuration- $(\mathrm{Be}-\mathrm{Be})_{\mathrm{Ga}}$. The strong bond between the two Be atoms in the split-interstitial defect gives a highfrequency stretch mode. It is likely that this is one of the two lines at 1055 and $1063 \mathrm{~cm}^{-1}$ observed by infrared spectroscopy, while the other simply represents a modified version of the defect where one of the neighboring $\mathrm{N}$ atoms is replaced by an $\mathrm{O}$ atom (the material has a high oxygen content). It is 




FIG. 5. Calculated LVM's for $\mathrm{Be}_{\mathrm{Ga}},(\mathrm{Be}-\mathrm{Be})_{\mathrm{Ga}}$, and $\mathrm{Be}_{\mathrm{Ga}}-\mathrm{O}_{\mathrm{N}}$ in $\mathrm{GaN}$ compared with modes observed by infrared spectroscopy (Ref. 42).

also quite possible that there is a very similar metastable variant of the $(\mathrm{Be}-\mathrm{Be})_{\mathrm{Ga}}$ defect that we have overlooked. The calculations also predict that there are two lower-frequency stretch-bend modes. However, an observed mode at $789 \mathrm{~cm}^{-1}$ appears to be due to a different defect for which there is no model at present, and it is only a coincidence that the lower-frequency modes of the split-interstitial are similar to this. One suggestion has been that it is due to $\mathrm{Be}_{\mathrm{Ga}}-\mathrm{O}_{\mathrm{N}}$ defects, but the calculations predict that the frequencies of its modes are far too low to explain the line seen in the experiments. However, another way to view the variant of the $(\mathrm{Be}-\mathrm{Be})_{\mathrm{Ga}}$ that is perturbed by an $\mathrm{O}_{\mathrm{N}}$ neighbor is as $\mathrm{Be}_{\mathrm{Ga}}-\mathrm{O}_{\mathrm{N}}$ plus $\mathrm{Be}_{i}$. The defect might form when an interstitial $\mathrm{Be}$ atom migrating though a crystal encounters a $\mathrm{Be}_{\mathrm{Ga}}-\mathrm{O}_{\mathrm{N}}$ defect instead of a single, substitional $\mathrm{Be}_{\mathrm{Ga}}$.

Finally, there remains one intriguing observation, not mentioned previously, that may prove to be crucial to understanding why Be-doped GaN is semi-insulating. Positron annihilation experiments find that the material contains a significant concentration of vacancylike traps where the positron lifetime is $210 \pm 10 \mathrm{ps}^{49,50}$ This figure is very slightly less than the measured lifetime of $V_{\mathrm{Ga}}(235 \pm 5 \mathrm{ps})$ and much longer than in the bulk crystal (160 1 ps). It is very unlikely that the defects are $V_{\mathrm{Ga}}$ : in semi-insulating material $\mu_{e}$ is near midgap, so the formation energy is prohibitively high. ${ }^{51,52,6} V_{\mathrm{N}}$ can also be excluded for the reasons mentioned in the Introduction. Preliminary results from work in progress suggest that the defect might be a nitrogen vacancy with one or more substitional beryllium atoms neighboring it. The Be atoms move away from the empty nitrogen site into a more planar configuration, thus increasing the open volume.

\section{ACKNOWLEDGMENTS}

This research has been supported in part the Academy of Finland through its Centers of Excellence Program 20002005. Computer resources have been provided by the Center for Scientific Computing in Finland (CSC) and Computer Services for Academic Research in the United Kingdom (CSAR). C.J.F. and R.J. wish to thank the Engineering and Physical Sciences Research Council (EPSRC), UK, for its financial support at the University of Exeter (Contract No. GR/K05528). S.O. thanks VR in Sweden for providing financial support. The authors would also like to express their thanks in particular to K. Saarinen, B. Clerjaud, Young Joo Lee, and P. Lehtinen for helpful discussions.
*Present address: School of Physics, University of Exeter, Exeter, EX4 4QL, United Kindom. Electronic address: C.D.Latham@ex.ac.uk

${ }^{1}$ E. R. Glaser, T. A. Kennedy, K. Doverspike, L. B. Rowland, D. K. Gaskill, J. A. Freitas, Jr., M. Asif Khan, D. T. Olson, J. N. Kuznia, and D. K. Wickenden, Phys. Rev. B 51, 13326 (1995).

${ }^{2}$ D. M. Hofmann, D. Kovalev, G. Steude, B. K. Meyer, A. Hoffmann, L. Eckey, R. Heitz, T. Detchprom, H. Amano, and I. Akasaki, Phys. Rev. B 52, 16702 (1995).

${ }^{3}$ T. Suski, P. Perlin, H. Teisseyre, M. Leszczyński, I. Grzegory, J. Jun, M. Boćkowski, S. Porowski, and T. D. Moustakas, Appl. Phys. Lett. 67, 2188 (1995).

${ }^{4}$ F. K. Koschnick, K. Michael, J.-M. Spaeth, B. Beaumont, and P. Gibart, Phys. Rev. B 54, R11 042 (1996).

${ }^{5}$ J. Neugebauer and C. G. Van de Walle, Appl. Phys. Lett. 69, 503 (1996)

${ }^{6}$ T. Mattila and R. M. Nieminen, Phys. Rev. B 55, 9571 (1997).

${ }^{7}$ X. Zhang, P. Kung, and M. Razeghi, in Gallium Nitride and Re- lated Materials, edited by R. D. Dupuis, F. A. Ponce, J. A. Edmond, and S. Nakamura, Mater. Res. Soc. Symp. Proc. No. 395 (World Scientific, Singapore, 1995), p. 625.

${ }^{8}$ W. Götz, N. Johnson, J. Walker, D. P. Bour, H. Amano, and I. Akasaki, in Proceedings of the 6th International Conference on SiC and Related Materials, Kyoto, Japan, 1995, edited by S. Nakashima, H. Matsunami, S. Yoshida, and H. Harima, Inst. Phys. Conf. Ser. No. 142 (Institute of Physics, London, 1996), p. 1031.

${ }^{9}$ W. Kim, A. Salvador, A. E. Botchkarev, O. Aktas, S. N. Mohammad, and H. Morcoç, Appl. Phys. Lett. 69, 559 (1996).

${ }^{10}$ K. Saarinen et al., Phys. Rev. Lett. 79, 3030 (1997).

${ }^{11} \mathrm{~K}$. Saarinen, in III-V Nitride Semiconductors: Electrical, Structural and Defect Properties, edited by M. O. Manasreh (Elsevier, Amsterdam, 2000), p. 109.

${ }^{12}$ W. Götz, N. M. Johnson, J. Walker, D. P. Bour, and R. A. Street, Appl. Phys. Lett. 68, 667 (1996).

${ }^{13}$ B. Clerjaud, D. Côte, A. Lebkiri, C. Naud, J. M. Baranowski, K. 
Pakula, D. Wasik, and T. Suski, Phys. Rev. B 61, 8238 (2000).

${ }^{14}$ J. Neugebauer and C. G. Van de Walle, Phys. Rev. Lett. 75, 4452 (1995).

${ }^{15}$ W. Götz, N. M. Johnson, C. Chen, H. Liu, C. Kuo, and W. Imler, Appl. Phys. Lett. 68, 3144 (1996).

${ }^{16}$ C. Bozdog et al., Phys. Rev. B 59, 12479 (1999).

${ }^{17}$ K. H. Chow, G. D. Watkins, A. Usui, and M. Mizuta, Phys. Rev. Lett. 8, 2761 (2000).

${ }^{18}$ W. M. Chen, I. A. Buyanova, M. Wagner, B. Monemar, J. L. Lindström, H. Amano, and I. Akasaki, Phys. Rev. B 58, R13 351 (1998)

${ }^{19}$ J. Neugebauer and C. G. Van de Walle, J. Appl. Phys. 85, 3003 (1999).

${ }^{20}$ C. G. Van de Walle, S. Limpijumnong, and J. Neugebauer, Phys. Rev. B 63, 245205 (2001).

${ }^{21}$ F. Mireles and S. E. Ulloa, Phys. Rev. B 58, 3879 (1998).

${ }^{22}$ B. Pödör, Semicond. Sci. Technol. 11, L827 (1996).

${ }^{23} \mathrm{R}$. Jones and P. R. Briddon, The ab initio Cluster Method and the Dynamics of Defects in Semiconductors, Vol. 51A of Semiconductors and Semimetals (Academic, Boston, 1998), Chap. 6.

${ }^{24}$ J. Coutinho, R. Jones, P. R. Briddon, and S. Öberg, Phys. Rev. B 62, 10824 (2000).

${ }^{25}$ C. D. Latham, R. Jones, S. Öberg, and P. R. Briddon, Phys. Rev. B 63, 155202 (2001).

${ }^{26}$ N. Troullier and J. L. Martins, Phys. Rev. B 43, 1993 (1991).

${ }^{27}$ S. G. Louie, S. Froyen, and M. L. Cohen, Phys. Rev. B 26, 1738 (1982)

${ }^{28}$ H. J. Monkhorst and J. D. Pack, Phys. Rev. B 13, 5188 (1976).

${ }^{29}$ M. J. P. Musgrave and J. A. Pople, Proc. R. Soc. London, Ser. A 268, 474 (1962).

${ }^{30}$ R. Jones, J. Phys. C 20, L271 (1987).

${ }^{31}$ S. B. Zhang and J. E. Northrup, Phys. Rev. Lett. 67, 2339 (1991).

${ }^{32}$ S. Pöykkö, M. J. Puska, and R. M. Nieminen, Phys. Rev. B 53, 3813 (1996).

${ }^{33}$ T. Mattila and R. M. Nieminen, Phys. Rev. B 54, 16676 (1996).
${ }^{34}$ L. Kleinman, Phys. Rev. B 24, 7412 (1981).

${ }^{35}$ I. N. Przhevalskii, S. Y. Karpov, and Y. N. Makarov, MRS Internet J. Nitride Semicond. Res. 3, 1 (1998).

${ }^{36}$ M. W. Chase, J. Phys. Chem. Ref. Data Monogr. 9, 1 (1998).

${ }^{37}$ Y. J. Lee and R. M. Nieminen (unpublished).

${ }^{38}$ C. H. Park and D. J. Chadi, Phys. Rev. B 55, 12995 (1997).

${ }^{39}$ V. Fiorentini, F. Bernardini, A. Bosin, and D. Vanderbilt, in The Physics of Semiconductors, edited by M. Scheffler and R. Zimmermann (World Scientific, Singapore, 1996), pp. 2877-2880.

${ }^{40}$ F. Bernardini, V. Fiorentini, and A. Bosin, Appl. Phys. Lett. 70, 2990 (1997).

${ }^{41}$ W. Götz, R. S. Kern, C. H. Chen, H. Liu, D. A. Steigerwald, and R. M. Fletcher, Mater. Sci. Eng., B 59, 211 (1999).

${ }^{42}$ B. Clerjaud (private communication). The Be-doped GaN used for these experiments was grown using a high-pressure, hightemperature method by UNIPRESS, Warsaw, Poland.

${ }^{43}$ A. Kaschner, H. Siegle, A. Hoffmann, C. Thomsen, U. Birkle, S. Einfeldt, and D. Hommel, MRS Internet J. Nitride Semicond. Res. 4S1, Ge.57 (1999).

${ }^{44}$ A. Kaschner, H. Siegle, G. Kaczmarczyk, M. Strassburg, A. Hoffmann, C. Thomsen, U. Birkle, S. Einfeldt, and D. Hommel, Appl. Phys. Lett. 74, 3281 (1999).

${ }^{45}$ H. Harima, T. Inoue, S. Nakashima, M. Ishida, and M. Taneya, Appl. Phys. Lett. 75, 1383 (1999).

${ }^{46}$ H. Harima, T. Inoue, Y. Sone, S. Nakashima, M. Ishida, and M. Taneya, Phys. Status Solidi B 216, 789 (1999).

${ }^{47}$ J. Menniger, U. Jahn, O. Brandt, H. Yang, and K. Ploog, Phys. Rev. B 53, 1881 (1996).

${ }^{48}$ C. Wetzel, H. Amano, I. Akasaki, J. W. Ager III, I. Grzegory, M. Topf, and B. K. Meyer, Phys. Rev. B 61, 8202 (2000).

${ }^{49}$ K. Saarinen (private communication).

${ }^{50}$ K. Saarinen, V. Ranki, T. Suski, M. Bockowski, and I. Grzegory, J. Cryst Growth 246, 281 (2002).

${ }^{51}$ J. Neugebauer and C. G. Van de Walle, Phys. Rev. B 50, 8067 (1994).

${ }^{52}$ P. Bogusławski, E. L. Briggs, and J. Bernholc, Phys. Rev. B 51, 17255 (1995). 